\title{
Comparing efficacy and survivals of initial treatments for elderly patients with newly diagnosed multiple myeloma: a network meta- analysis of randomized controlled trials
}

\author{
This article was published in the following Dove Press journal: \\ OncoTargets and Therapy \\ 22 December 2016 \\ Number of times this article has been viewed
}

\section{Xiaoping Liu',* \\ Jiarui Chen ${ }^{2, *}$ \\ Yuncen $\mathrm{A} \mathrm{He}^{3}$ \\ Xiangyu Meng ${ }^{\prime}$ \\ Kaili $\mathrm{Li}^{4}$ \\ Colin $\mathrm{K} \mathrm{He}^{5}$ \\ Shangqin $\mathrm{Liu}^{4}$}

'Center for Evidence-Based and Translational Medicine, Zhongnan Hospital of Wuhan University, ${ }^{2}$ School of Basic Medical Science, Wuhan University, Wuhan, Hubei, People's Republic of China; ${ }^{3}$ School of Medicine, Case Western Reserve University, Cleveland, OH, USA; ${ }^{4}$ Department of Hematology, Zhongnan Hospital of Wuhan University, Wuhan, Hubei, People's Republic of China; ${ }^{5}$ Orient Healthcare, New York, NY, USA

*These authors contributed equally to this work
Correspondence: Shangqin Liu Department of Hematology, Zhongnan Hospital of Wuhan University, Donghu Road 169, Wuhan, Hubei, 43007I, People's Republic of China Emailwb001458@whu.edu.cn

Colin $\mathrm{K} \mathrm{He}$

StegoTech LLC, 422 Lynrsoe CT, King of Prussia, PA 19406, USA

Email statqcbi@aliyun.com
Objective: The aim of this study was to evaluate the efficacy and clinical outcome of initial therapies for elderly patients with multiple myeloma (MM).

Methods: Randomized controlled trials (RCTs) were obtained through a comprehensive search. Response rate, progression-free survival (PFS) and overall survival (OS) were the interested outcome measures. Network meta-analysis (NMA) using graph theory methodology to construct an NMA model, and sensitivity analysis were performed.

Results: Nineteen RCTs containing 7,235 participants and 17 treatments were included in the NMA. As compared to the classic melphalan plus prednisone (MP) regimen, the majority of other initial regimens showed higher rates of complete response/near complete response, overall response rate (ORR) and better PFS as well as OS. These four outcomes favored the two lenalidomide plus dexamethasone regimens (continuous lenalidomide and 18 cycles of lenalidomide plus dexamethasone), especially continuous lenalidomide plus dexamethasone regimen, over the majority of other regimens including the two established standard treatments (MP plus thalidomide or bortezomib) for elderly patients with newly diagnosed MM.

Conclusion: Continuous lenalidomide plus dexamethasone ranked as the best regimen in terms of ORR and OS for the treatment of elderly patients with newly diagnosed MM.

Keywords: multiple myeloma, previously untreated, elderly patients, initial therapies, network meta-analysis

\section{Introduction}

Multiple myeloma (MM), a plasma cell neoplasm characterized by the production of monoclonal immunoglobulin, is the second most common hematological malignancy. ${ }^{1}$ With the increased life expectancy of the normal population, more than two-thirds of MM patients are first diagnosed at the age of $>65$ years. ${ }^{2}$ High-dose therapy (HDT) plus autologous stem cell transplantation (ASCT) and the introduction of novel agents, such as bortezomib, carfizomib, thalidomide, lenalidomide and pomalidomide, have substantially improved response rate and prognosis for MM patients $<65$ years; ${ }^{3,4}$ however, the outcome of patients who are $>65$ years and generally considered unsuitable for HDT-ASCT remains poor. These patients usually need milder therapies.

Melphalan plus prednisone (MP) regimen has long been considered the standard care for elderly MM patients, ${ }^{5}$ and the application of the abovementioned novel agents has enriched the treatments for elderly MM patients. Nowadays, MP 
plus thalidomide/lenalidomide, MP plus bortezomib and lenalidomide/thalidomide plus dexamethasone are the widely used regimens for elderly MM patients. ${ }^{3}$ Moreover, most of the Phase III randomized controlled trials (RCTs) compared MP regimen with MP-based regimens that include novel agents, yet few RCTs take regimens with novel agents into direct comparison. For this reason, network meta-analysis (NMA) of RCTs comparatively evaluating the response rate (complete response/near complete response [CR/nCR] and overall response rate [ORR]) and survival prognosis (progression-free survival [PFS] and overall survival [OS]) of the initial treatment for elderly MM patients is of necessity and interest. The NMA was performed in accordance with the Preferred Reporting Items for Systematic Reviews and Meta-Analyses (PRISMA) statement. ${ }^{6}$

\section{Methods}

\section{Data sources}

We carried out a comprehensive literature search in existing databases such as PubMed, Embase, and the Cochrane Library and the Science Citation Index as well as relevant websites (eg, http://www.controlledtrials.com/ and https:// www.ClinicalTrials.gov/ct) aiming at identifying the potentially eligible RCTs for our analysis. Data from conference proceedings of the American Society of Hematology (2000-2015), the American Society of Clinical Oncology (2000-2015) and the European Hematology Association were also collected. Literature searches were last updated on April 20, 2016. The keywords utilized were "multiple myeloma OR plasmacytoma", "newly diagnosed OR firstly diagnosed OR previously untreated", "older patients OR elderly patients OR transplant ineligible OR unsuitable for transplantation" and "initial treatment OR initial therapy". In addition, potentially relevant studies in the references of the trials were identified and other published systemic reviews and practice guidelines were examined as well. We selected potentially eligible studies through reviewing the titles and abstracts from the results of our search strategy. Furthermore, full-text articles were independently reviewed by two authors to ensure the studies meet the following inclusion criteria: 1) the studies were RCTs; 2) the participants were elderly patients with newly diagnosed MM who were unsuitable for HDT and 3) interventions were initial therapy for MM patients. Studies that did not focus on elderly patients or report the key end points $(\mathrm{CR} / \mathrm{nCR}$, ORR, PFS and OS) of our interest were excluded. According to the Jadad scale including the reporting of the randomization method, blinding scores and completeness of follow-up, the maximal score for an included study was 5 and studies were classified on the basis of quality as high (score: $3-5$ ) versus low (score: $0-2)^{7}$

\section{Outcome measures}

The goal of this NMA is to evaluate the effect of initial therapy for elderly MM patients. The key end points of this review are PFS (calculated from the time of randomization until the date of progression or death from any cause during treatment or until data censoring at the last date at which the patient was known to be progression free), OS (measured from the time of randomization until the date of death from any cause or until data censoring at the last date at which the patient was known to be alive), $\mathrm{CR} / \mathrm{nCR}$ and ORR (evaluated with reference to the international uniform response criteria). ${ }^{8}$

\section{Data extraction}

Relevant studies were investigated through full-text review, and only those that met all the inclusion criteria were included in the final analysis. A predesigned data extraction form involving baseline characteristics, outcomes and number of events was utilized in the data extraction process, which was conducted independently by Xiaoping Liu and Jiarui Chen. Any discrepancies between the two investigators at the screening or data extraction stage were resolved by discussion.

\section{Statistical analysis}

We utilized the R Project software, version 3.2.2 (www.rproject.org) and Stata 13.0 (StataCorp, College Station, TX, USA) to perform the statistical analysis. For the end points of $\mathrm{CR} / \mathrm{nCR}$ and ORR, the natural log of relative risk (RR) and its standard error were applied to evaluate the effect of the initial treatments in elderly MM patients. Meanwhile, the natural log of hazard ratio (HR) and its standard error were applied to evaluate the survival data of these patients. In case the unavailability of any HR and its $95 \%$ confidence interval (CI) was detected in any studies, we calculated the HRs and 95\% CIs with the method introduced by Tierney et al. ${ }^{9}$ We adopted $I^{2}$ statistic to quantify heterogeneity among the studies. When low heterogeneity was detected (the value of $I^{2}$ was $<50 \%$ ), a fixed-effects model was preferred for the meta-analysis. Conversely, if the value of $I^{2}$ was detected to be $>50 \%$, we applied the random-effects model instead. Meanwhile, a loop-specific approach was applied to evaluate the consistency assumption in each closed loop of the network. In each loop, we estimated the inconsistency factor 
(IF) as the absolute difference between direct and indirect estimates for one of the comparisons in the loop. The IF is the $\log$ of two odds ratios (RoR) from direct and indirect evidence in the loop. RoR values close to 1 indicate that the two sources are in agreement. ${ }^{10,11}$ The graph-theoretical method that exploits the analogy between treatment networks and electrical networks was used to construct an NMA model accounting for the correlated treatment effects in multiarm studies. ${ }^{12}$ This has been found to be equivalent to the frequentist approach to NMA and can be implemented via the R package "netmeta" (version 0.8-0). ${ }^{13}$ In addition, using the $P$-value recommended by Rücker and Schwarzer, ${ }^{14}$ we estimated the probability that each treatment was the best regimen, the second best and so on.

\section{Results}

\section{Literature search results}

A total of 13,155 articles were obtained through our literature search, of which 10,932 were excluded since they were not clinical trials. After reviewing the titles and abstracts, 28 potentially relevant RCTs were considered well worth full-text reading. Finally, the following nine articles were excluded: four articles were updated analysis of three other trials, two articles did not focus on elderly populations, one article was a duplicate report, one article did not address the initial therapy for elderly patients with newly diagnosed MM and one article alternated the schemes of induction therapy in different ways. Hence, a total number of 19 studies were eventually included in this NMA (Figure 1). ${ }^{15-33}$

\section{Description of included trials}

A total number of 7,235 participants were randomly assigned in these included trials with 36-547 participants per arm. The trials assessed a total of 17 interventions or control conditions, which were grouped into 23 pairwise comparisons (Figure 2). Most trials included two arms $(n=14)$, but five trials included three arms. The most common pairwise comparison was MP plus thalidomide (MPT) versus MP $(n=6)$. It is worth noting that patients who completed the planned MPT cycles received maintenance therapy with oral thalidomide until disease progression in four trials (MPT-T), whereas in other two trials, patients ended their planned MPT without any maintenance therapy administrated. Other regimens used in these included trials were as follows: cyclophosphamide plus thalidomide plus dexamethasone (CTD) regimen in one trial, cyclophosphamide plus prednisone plus lenalidomide (CPR)

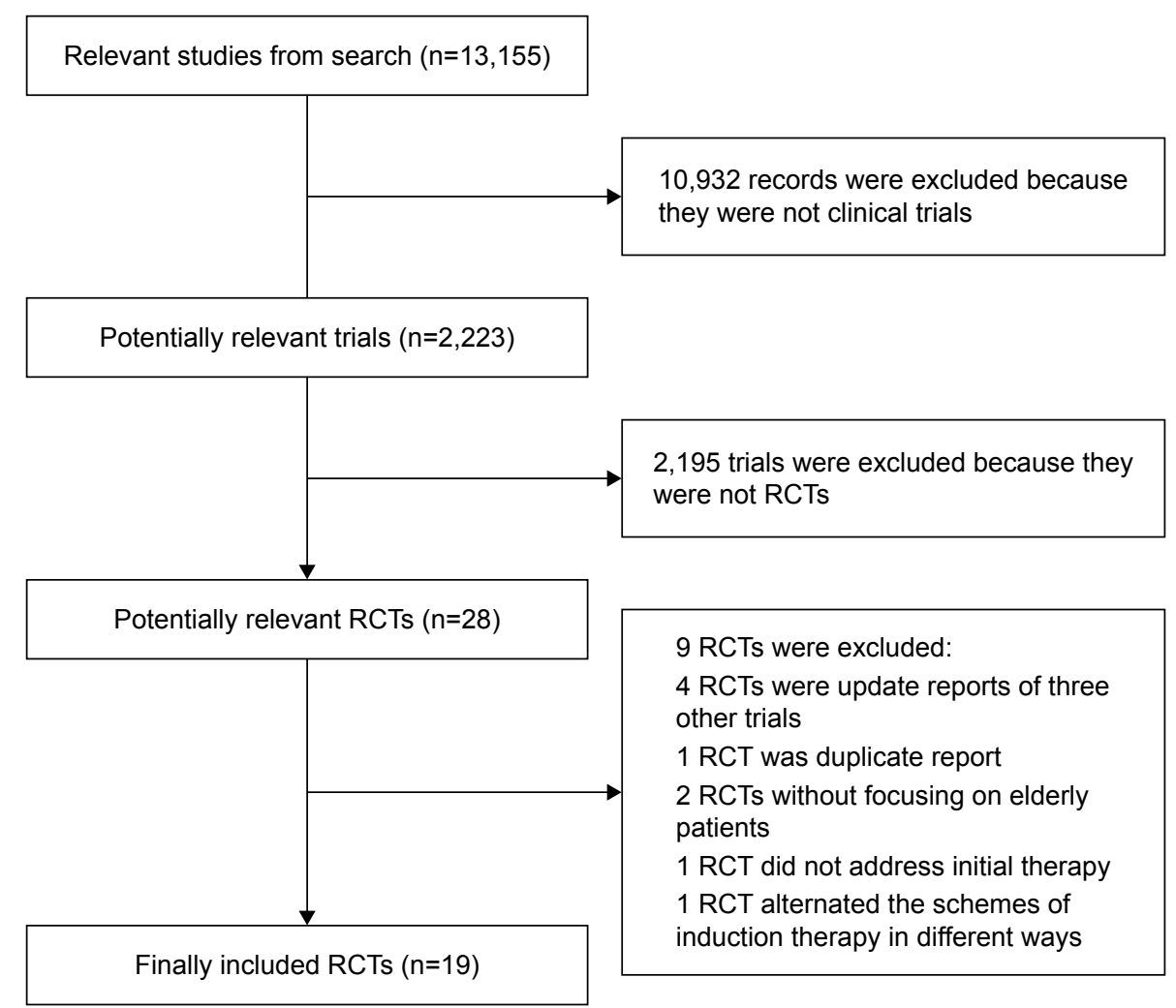

Figure I Flow diagram showing the publication selection process. Abbreviation: RCTs, randomized controlled trials. 


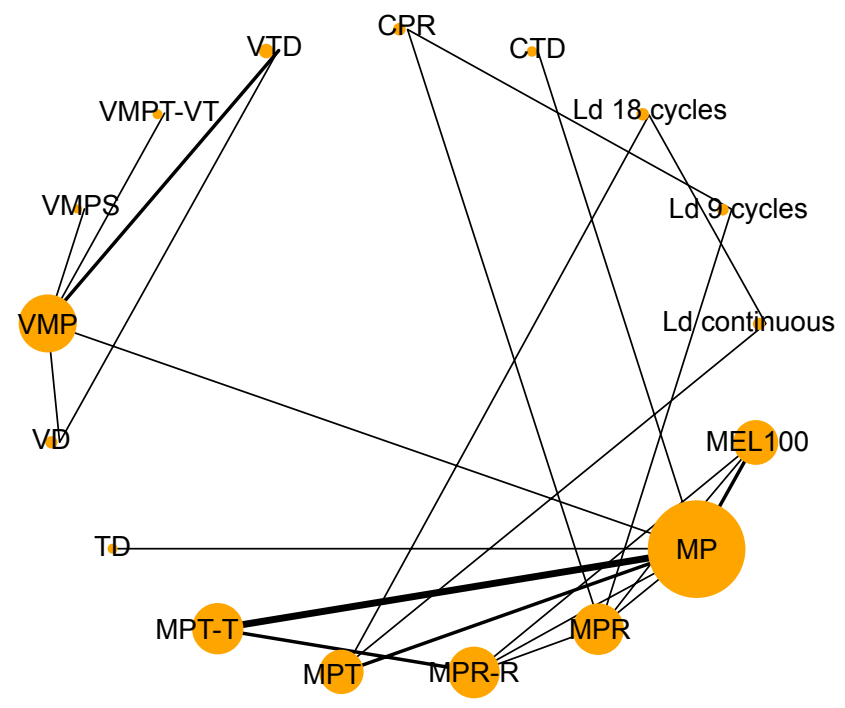

Figure 2 Network plot of initial treatments included in this network meta-analysis. Notes: Circles represent the intervention as a node in the network, lines represent direct comparisons using randomized controlled trials (RCTs) and the thickness of lines corresponds to the number of RCTs included in each comparison.

Abbreviations: CPR, cyclophosphamide plus prednisone and lenalidomide; CTD, cyclophosphamide plus thalidomide and dexamethasone; Ld 9 cycles, lenalidomide plus dexamethasone for 9 cycles; Ld 18 cycles, lenalidomide plus dexamethasone for 18 cycles; Ld continuous, lenalidomide plus dexamethasone in 28-day cycles until disease progression; MELI00, reduced-intensity stem cell transplantation using melphalan $100 \mathrm{mg} / \mathrm{m}^{2}$; MP, melphalan plus prednisone; MPR, MP plus lenalidomide; MPR-R, MPR regimen followed by lenalidomide maintenance therapy until a relapse or disease progression occurred; MPT, MP plus thalidomide; MPT-T, MPT cycles received maintenance therapy with oral thalidomide until disease progression in four trials; RCTs, randomized controlled trials; TD, thalidomide plus dexamethasone; VD, bortezomib plus dexamethasone; VMP, bortezomib plus melphalan plus prednisone; VMPS, VMP plus siltuximab; VMPTVT, VMP plus thalidomide that followed by maintenance with bortezomib and thalidomide; VTD, bortezomib plus TD.

in one trial, lenalidomide plus dexamethasone in 28-day cycles until disease progression (Ld continuous) and the same combination of lenalidomide plus dexamethasone for 18 cycles (Ld 18 cycles) in one trial, lenalidomide plus dexamethasone for 9 cycles ( $\mathrm{Ld} 9$ cycles) in one trial, reduced-intensity stem cell transplantation using melphalan $100 \mathrm{mg} / \mathrm{m}^{2}$ (MEL100) in two trials, thalidomide plus dexamethasone (TD) in one trial, bortezomib plus dexamethasone (VD) in one trial, bortezomib plus MP (VMP) in five trials, VMP plus siltuximab (VMPS) in one trial, VMP plus thalidomide that followed by maintenance with bortezomib plus thalidomide (VMPT-VT) in one trial, bortezomib plus TD (VTD) in two trials and MP plus lenalidomide (MPR) and the same combination of MPR regimen followed by lenalidomide maintenance therapy until a relapse or disease progression occurred (MPR-R) in two trials and three trials, respectively (Table S1). The methodological quality of each study assessed according to the Jadad scale is shown in Table S2. Demographic characteristics from each trial population, including median age, gender, types of $\mathrm{M}$ protein, International Staging System stage, median $\beta 2$-microglobulin and median albumin, are listed in Table S3.

\section{Response to treatment}

All 19 included RCTs reported the data of $\mathrm{CR} / \mathrm{nCR}$ and ORR for the NMA. For the $\mathrm{CR} / \mathrm{nCR}$, no statistically significant evidence of inconsistency was found (Figure S1A) and, according to the value of associated $I^{2}\left(I^{2}=18.6 \%\right)$, we selected the fixed-effects model to perform the analysis. As compared to MP, all the other regimens showed higher $\mathrm{CR} / \mathrm{nCR}$ rates. $\mathrm{Ld} 18$ cycles as well as Ld continuous were more efficacious than CPR, MEL100, MP, MPR, MPR-R, MPT, MPT-T and TD. Meanwhile, VMP, VMPS, VMPT and VTD had significantly higher rates of $\mathrm{CR} / \mathrm{nCR}$ than those resulted by CPR, MP, MPR, MPR-R, MPT-T and TD. VMPS was associated with higher $\mathrm{CR} / \mathrm{nCR}$ rates compared to VD and VMP. VTD was more efficacious compared to VD. No statistically significant difference could be found among VMPS, VMPT-VT, VTD, Ld 18 cycles and Ld continuous, and no significant difference could be found among MPR, MPR-R, MPT-T and TD (Figure 3A and Table S4). VMPS, Ld 18 cycles and Ld continuous ranked as the best three regimens for this outcome, and VMPS was ranked the best regimen for this outcome with relatively higher probability $(P$-value $=0.8711$, Table $\mathrm{S} 5)$. With respective to the end point ORR, the fixed-effects model was preferred according to the value of $I^{2}\left(I^{2}=0\right)$, and there was no evident inconsistency found in the network for this outcome (Figure $\mathrm{S} 1 \mathrm{~B}$ ). As with $\mathrm{CR} / \mathrm{nCR}$, all the regimens resulted in higher rates of ORR compared to MP. Ld 18 cycles and $\mathrm{Ld}$ continuous were more efficacious than CPR, Ld 9 cycles, MEL100, MPR, MPR-R, MPT, MPT-T and TD. VD, VMP, VMPS, VMPT and VTD had significantly higher rates of ORR than those resulted by CPR, Ld 9 cycles, MP, MPR, MPR-R, MPT-T and TD. VMPT-VT was associated with higher rates of ORR than VMP. As with $\mathrm{CR} / \mathrm{nCR}$, no evident difference was found in the other comparisons (Figure 3B and Table S6). Ld continuous, Ld 18 cycles, VMPT-VT and VMPS ranked as the best four regimens for this outcome, and $\mathrm{Ld}$ continuous was ranked the best regimen for this outcome with relatively higher probability $(P$-value $=0.9510, \underline{\text { Table S5 }})$.

\section{PFS}

A total number of 18 RCTs reported PFS-associated data for this NMA. Owning to the absence of PFS-associated data in the trial conducted by San Miguel et al, ${ }^{17}$ the whole network framework was split into two separated networks. According to the respective $I^{2}$ values $\left(I^{2}=73.6 \%\right.$ and $\left.73.5 \%\right)$, the random-effects model was preferred for both subnetworks and no evident inconsistency was found (Figure S1C). Ld continuous, MPR-R, MPT and MPT-T resulted in better 

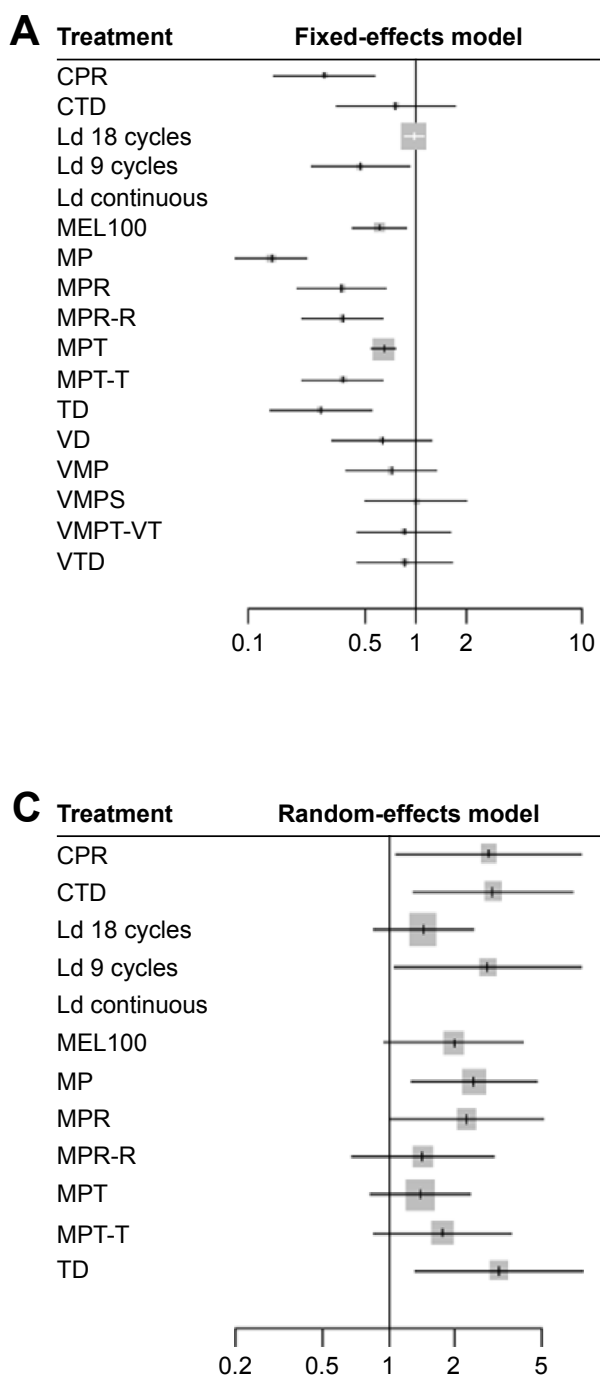

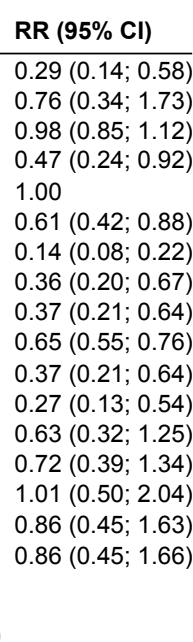

$0.29(0.14 \cdot 0.58)$

$0.76(0.34 ; 1.73)$

$98(0.85 ; 1.12)$

1.00

$0.14(0.08 ; 0.22)$

$0.65(0.55 ; 0.76)$

$7(0.21 ; 0.64)$

$.27(0.13 ; 0.54)$

$63(0.32 ; 1.25)$

$1.01(0.50 ; 2.04)$

$0.86(0.45 ; 1.63)$

$0.86(0.45 ; 1.66)$

B
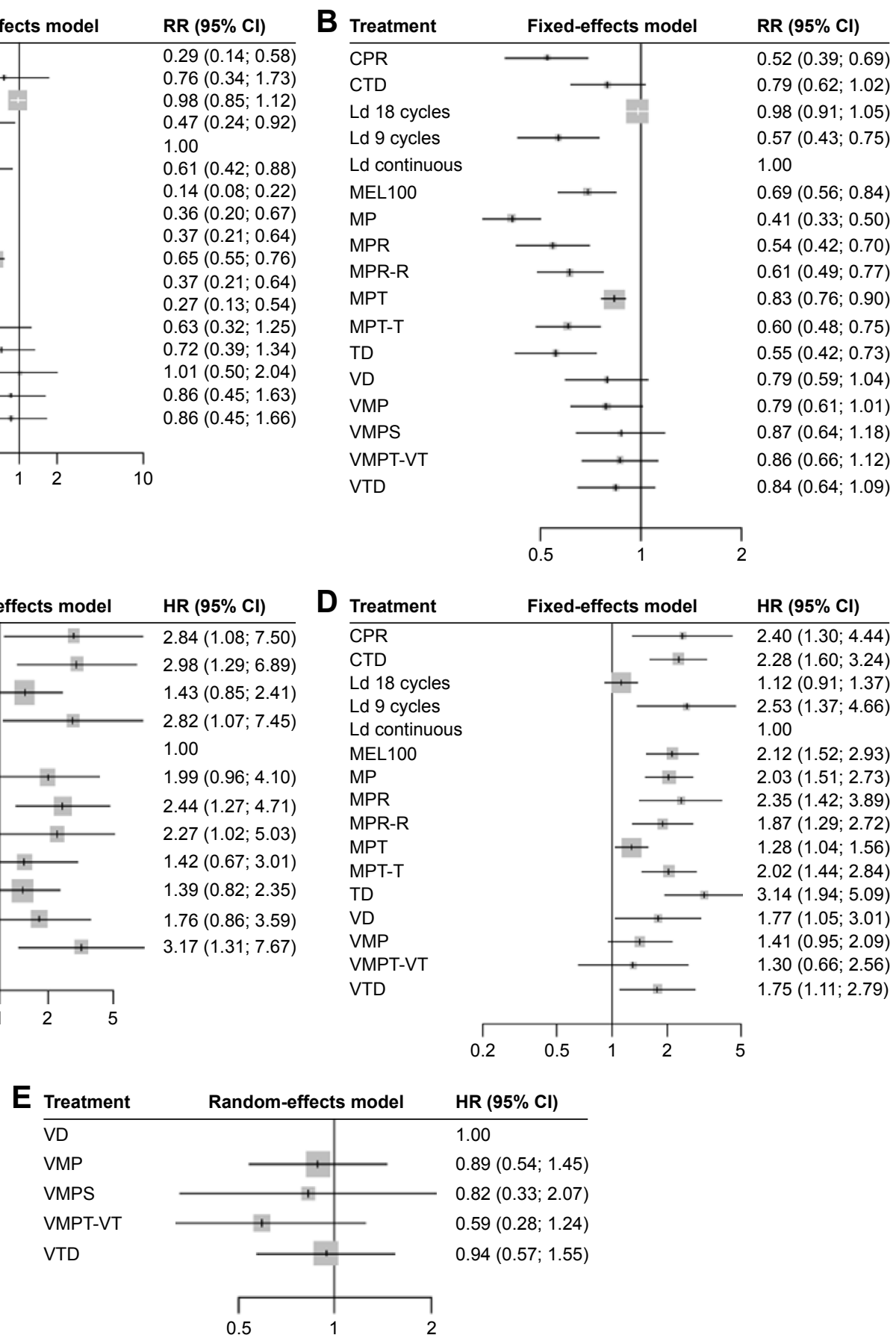

Figure 3 Differences in CR/nCR, ORR, PFS and OS between Ld continuous or VD regimen versus other initial regimens.

Notes: (A) Network analysis of CR/nCR, (B) network analysis of ORR, (C) subnetwork analysis of PFS, (D) network analysis of OS and (E) subnetwork analysis of PFS Abbreviations: $\mathrm{Cl}$, confidence interval; CPR, cyclophosphamide plus prednisone and lenalidomide; CR/nCR, complete response/near complete response; CTD, cyclophosphamide plus thalidomide and dexamethasone; HR, hazard ratio; Ld 9 cycles, lenalidomide plus dexamethasone for 9 cycles; Ld I8 cycles, lenalidomide plus dexamethasone for 18 cycles; Ld continuous, lenalidomide plus dexamethasone in 28-day cycles until disease progression; MELI 00, reduced-intensity stem cell transplantation using melphalan $100 \mathrm{mg} / \mathrm{m}^{2}$; MP, melphalan plus prednisone; MPR, MP plus lenalidomide; MPR-R, MPR regimen followed by lenalidomide maintenance therapy until a relapse or disease progression occurred; MPT, MP plus thalidomide; MPT-T, MPT cycles received maintenance therapy with oral thalidomide until disease progression in four trials; ORR, overall response rate; OS, overall survival; PFS, progression-free survival; RR, relative risk; TD, thalidomide plus dexamethasone; VD, bortezomib plus dexamethasone; VMP, bortezomib plus melphalan plus prednisone; VMPS, VMP plus siltuximab; VMPT-VT, VMP plus thalidomide that followed by maintenance with bortezomib and thalidomide; VTD, bortezomib plus TD.

PFS than MP. The PFS favored MPR-R and MPT over MP, TD and CTD. Ld continuous resulted in better PFS than CPR, CTD, Ld 9 cycles, MP, MPR and TD. There was no evident difference in the other comparisons in this subnetwork (Figure 3C and Table S7a). Meanwhile, in the other subnetworks, no significant difference could be found among regimens VD, VMP, VMPS, VMPT-VT and VTD in terms of PFS (Figure $3 \mathrm{E}$ and Table S7b). Ld continuous 
$(P$-value $=0.9506)$ and VMPT-VT $(P$-value $=0.8759)$ ranked as the best for the two subnetworks (Table S5).

\section{OS}

Except for one trial conducted by San Miguel et al, ${ }^{15}$ OS was reported in $18 \mathrm{RCTs}$ for this network. According to the value $I^{2}\left(I^{2}=40 \%\right)$, the fixed-effects model was preferred and there was no evident inconsistency found in the network (Figure S1D). Ld 18 cycles, Ld continuous, MP, MPR-R, MPT, MPT-T, VMP, VMPT-VT and VTD were associated with significant superiority when compared with TD in terms of OS. Ld 18 cycles resulted in better OS than CPR, CTD, Ld 9 cycles, MEL100, MP, MPR, MPR-R, MPT-T and TD. Ld continuous resulted in better OS than CPR, CTD, MEL100, MP, MPR, MPR-R, MPT, MPT-T, TD, VD and VTD. VMP was associated with better OS than TD, MPT-T, MPR, MP, MEL100 and CTD. There was no significant difference among VD, VMP, VMPT-VT and VTD for the outcome of OS (Figure 3D and Table S8). Ld 18 cycles and Ld continuous ranked as the best two regimens for OS, and $\mathrm{Ld}$ continuous ranked as the best regimen for this outcome with relatively higher probability $(P$-value $=0.9384$, Table S5 $)$.

\section{Sensitivity analysis}

We performed sensitivity analysis of studies reporting on $>100$ patients in each arm and studies containing nontransplant therapy; thus, four trials were excluded for sensitivity analysis. ${ }^{15,21,25,27}$ The result indicated that Ld 18 cycles and Ld continuous had higher rates of $\mathrm{CR} / \mathrm{nCR}$ than MP and MPT. VMPT-VT and VTD had higher rates of CR/ nCR than CPR, MP, MPR, MPR-R, MPT-T, TD and VD (Table S9). Meanwhile, VMP, VMPT-VT and VTD were associated with higher rates of ORR than CPR, Ld 9 cycles, MP, MPR, MPR-R, MPT-T and TD. VMPT-VT had higher rates of ORR than VMP. Ld 18 cycles and Ld continuous were more efficacious than CPR, Ld 9 cycles, MP, MPR, MPR-R, MPT, MPT-T and TD in terms of ORR. As compared to MP, all the other regimens demonstrated higher rates of ORR (Table S10). For the outcome of PFS and OS, PFS favored Ld continuous over TD, MP and CTD. Ld continuous, MPR-R and MPT-T had better PFS compared to MP (Table S11). Meanwhile, Ld continuous, Ld 18 cycles, VMP and MPT have better OS than MP. Furthermore, Ld continuous demonstrated better OS than CPR, CTD, Ld 9 cycles, MP, MPR, MPR-R, MPT, MPT-T and TD. OS favor VMP over CTD, MP, MPR, MPR-R, MPT-T and TD (Table S12). Ld continuous ranked as the best regimen for the outcome of ORR and OS ( $P$-values 0.9189 and 0.9237 , respectively).

\section{Discussion}

Treatments for elderly patients with newly diagnosed MM have changed dramatically with the introduction of novel agents including proteasome inhibitors (bortezomib) and immunomodulatory drugs (thalidomide and lenalidomide), and combined chemotherapies containing these novel agents varied. ${ }^{5}$ Although several trials and standard pairwise metaanalysis had been conducted to compare these combined chemotherapies for elderly MM patients, ${ }^{15,17,20,34}$ the most efficacious and tolerable regimens remain unclear. NMA, which can take both direct evidence within clinical trials and indirect evidence across clinical trials into a whole comparison and facilitate indirect comparisons of multiple inventions that have not been compared in head-to-head clinical trials, has been applied to characterize the optimal choice in many diseases including MM. ${ }^{35-38}$

Several clinical trials and meta-analysis suggested that MPT regimen had better outcome than traditional MP, which was confirmed in our NMA, and the majority of regimens studied in our NMA showed superiority compared to MP. For years, MPT and VMP were established as standard therapies for elderly MM patients unsuitable for HDTs. However, the introduction of lenalidomide plus dexamethasone might change this pattern. ${ }^{39}$ Benboubker et $\mathrm{al}^{20}$ demonstrated that response rates were higher in Ld continuous (75\%) and Ld 18 cycles $(73 \%)$ groups when compared with MPT group (62\%), and Ld continuous resulted in a significant improvement in PFS when compared with MPT $(\mathrm{HR}=0.72$, $P<0.001)$ and $\mathrm{Ld} 18$ cycles $(\mathrm{HR}=0.7, P<0.001)$. Similarly, our indirect comparisons suggested that continuous lenalidomide plus dexamethasone showed superiority compared to $\mathrm{MPT}\left(\mathrm{RR}_{\mathrm{CR} / \mathrm{nCR}}=1.55,95 \% \mathrm{CI}: 1.31-1.82 ; \mathrm{RR}_{\mathrm{ORR}}=1.21\right.$, 95\% CI: $\left.1.11-1.31 ; \mathrm{HR}_{\mathrm{OS}}=0.78,95 \% \mathrm{CI}: 0.64-0.96\right)$ and VMP $\left(\mathrm{RR}_{\mathrm{ORR}}=0.79,95 \% \mathrm{CI}: 0.61-1.01 ; \mathrm{HR}_{\mathrm{OS}}=1.41,95 \%\right.$ CI: 0.95-2.09), and the similar trend could be found when comparing Ld 18 cycles with MPT $\left(\mathrm{RR}_{\mathrm{CR} / \mathrm{nCR}}=0.66,95 \% \mathrm{CI}\right.$ : 0.56-0.78; $\mathrm{RR}_{\mathrm{ORR}}=0.85,95 \% \mathrm{CI}: 0.58-0.72 ; \mathrm{HR}_{\mathrm{OS}}=1.14$, 95\% CI: $0.94-1.39)$ and VMP $\left(\mathrm{RR}_{\mathrm{CR} / \mathrm{nCR}}=1.39,95 \% \mathrm{CI}\right.$ : 0.74-2.58; $\mathrm{RR}_{\mathrm{ORR}}=1.27,95 \% \mathrm{CI}: 0.99-1.63 ; \mathrm{HR}_{\mathrm{OS}}=0.71$, 95\% CI: 0.48-1.05). For the outcomes CR/nCR, ORR and OS, no significant difference could be found between Ld 18 cycles and 18 continuous, whereas Ld continuous had better PFS than Ld 18 cycles (HR $=0.70,95 \%$ CI: $0.48-1.08$ ), which was confirmed by a previous clinical trial. ${ }^{20}$ According to the $P$-values, bortezomib-based regimens (VD, VTD, VMP, VMPS and VMPT-VT) were also associated with promising antimyeloma activity, while these regimens had similar antimyeloma activities. Previous studies suggested 
that melphalan might be the best partner for bortezomibbased induction regimen in elderly patients with untreated MM when the efficacy, toxicities, and costs were taken into consideration. ${ }^{29,40}$

The NMA has limitations. 1) Owing to the definitions of adverse events in our included trials that differed significantly across the initial regimens, we could not perform a comprehensive analysis of adverse events, which prevented a balanced evaluation of both efficacy and tolerability for the initial treatments of elderly MM patients. Actually, in the FIRST trial ${ }^{20}$, the author demonstrated that both $\mathrm{Ld}$ continuous and Ld 18 cycles groups had lower rates of hematologic toxic events than the MPT group, peripheral sensory neuropathy was more common with MPT than with Ld continuous and Ld 18 cycles groups and the incidence of second primary cancers was low across treatment groups, but the incidence of grade 3 or 4 infection was increased with Ld continuous as compared with Ld 18 cycles or MPT (29\% vs $22 \%$ and $17 \%$ ) and $\mathrm{Ld}$ continuous was associated with an increase of 5 percentage points in the infection of any grade and an increase of 2 percentage points in thromboembolic events of grade 3 or 4 . Glucocorticoids might contribute partly to the long-term adverse events with $\mathrm{Ld}$ continuous, and lower doses of dexamethasone or the use of prednisone in the future studies might be of interest. ${ }^{20}$ 2) Many treatment pairs in the network were connected by a single study, and therefore, the results may be sensitive to changes in a single trial result. Therefore, the results should be interpreted with cautions and further studies are required to strengthen the NMA.

\section{Conclusion}

Our NMA demonstrated that the two lenalidomide plus dexamethasone initial treatments (18 cycles of lenalidomide plus dexamethasone and continuous lenalidomide plus dexamethasone), especially the continuous lenalidomide plus dexamethasone, resulted in better efficacy and prognosis for the elderly patients with MM.

\section{Acknowledgments}

This study was supported by the Research Fund from the National Natural Science Foundation of China (no 81272627). The abstract of this article was presented at the 2016 ASA Biopharmaceutical Section Regulatory-Industry Statistics Workshop as a roundtable discussion.

\section{Disclosure}

The authors report no conflicts of interest in this work.

\section{References}

1. Sabattini E, Bacci F, Sagramoso C, Pileri SA. WHO classification of tumours of haematopoietic and lymphoid tissues in 2008: an overview. Pathologica. 2010;102(3):83-87.

2. Broijl A, Sonneveld P. An update in treatment options for multiple myeloma in nontransplant eligible patients. Expert Opin Pharmacother. 2015;16(13):1945-1957.

3. Cottini F, Anderson K. Novel therapeutic targets in multiple myeloma. Clin Adv Hematol Oncol. 2015;13(4):236-248.

4. Liu X, He CK, Meng X, et al. Bortezomib-based vs non-bortezomibbased post-transplantation treatment in multiple myeloma patients: a systematic review and meta-analysis of Phase III randomized controlled trials. Onco Targets Ther. 2015;8:1459-1469.

5. Johnson TM. Optimal management of elderly patients with myeloma. Consult Pharm. 2014;29(7):434-438.

6. Hutton B, Salanti G, Caldwell DM, et al. The PRISMA extension statement for reporting of systematic reviews incorporating network meta-analyses of health care interventions: checklist and explanations. Ann Intern Med. 2015;162(11):777-784.

7. Jadad AR, Moore RA, Carroll D, et al. Assessing the quality of reports of randomized clinical trials: is blinding necessary? Control Clin Trials. 1996;17(1):1-12.

8. Durie BG, Harousseau JL, Miguel JS, et al. International uniform response criteria for multiple myeloma. Leukemia. 2006;20(9):1467-1473.

9. Tierney JF, Stewart LA, Ghersi D, Burdett S, Sydes MR. Practical methods for incorporating summary time-to-event data into meta-analysis. Trials. 2007;8:16.

10. Chaimani A, Higgins JP, Mavridis D, Spyridonos P, Salanti G. Graphical tools for network meta-analysis in STATA. PLoS One. 2013; 8(10):e76654.

11. Higgins JP, Jackson D, Barrett JK, Lu G. Ades AE4, White IR. Consistency and inconsistency in network meta-analysis: concepts and models for multi-arm studies. Res Synth Methods. 2012;3(2):98-110.

12. Rücker G. Network meta-analysis, electrical networks and graph theory. Res Syn Methods. 2012;3(4):312-324.

13. Rücker G, Schwarzer G, Krahn U, König J [webpage on the Internet]. netmeta: Network Meta-Analysis using Frequentist Methods. R package version 0.8-0. 2015. Available from: http://cran.at.r-project.org/web/ packages/netmeta/. Accessed November 25, 2016.

14. Rücker G, Schwarzer G. Ranking treatments in frequentist network meta-analysis works without resampling methods. BMC Med Res Methodol. 2015;15:58.

15. San-Miguel J, Bladé J, Shpilberg O, et al. Phase 2 randomized study of bortezomib-melphalan-prednisone with or without siltuximab (antiIL-6) in multiple myeloma. Blood. 2014;123(26):4136-4142.

16. Palumbo A, Bringhen S, Liberati AM, et al. Oral melphalan, prednisone, and thalidomide in elderly patients with multiple myeloma: updated results of a randomized controlled trial. Blood. 2008;112(8):3107-3114.

17. San Miguel JF, Schlag R, Khuageva NK, et al. Persistent overall Survival benefit and no increased risk of second malignancies with bortezomibmelphalan-prednisone versus melphalan-prednisone in patients with previously untreated multiple myeloma. J Clin Oncol. 2013;31(4): 448-455.

18. Mateos MV, Oriol A, Martínez-López J, et al. GEM2005 trial update comparing VMP/VTP as induction in elderly multiple myeloma patients: do we still need alkylators? Blood. 2014;124(12):1887-1893.

19. Morgan GJ, Davies FE, Gregory WM, et al; NCRI Haematological Oncology Study Group. Cyclophosphamide, thalidomide, and dexamethasone (CTD) as initial therapy for patients with multiple myeloma unsuitable for autologous transplantation. Blood. 2011;118(5):1231-1238.

20. Benboubker L, Dimopoulos MA, Dispenzieri A, et al; FIRST Trial Team. Lenalidomide and dexamethasone in transplant-ineligible patients with myeloma. N Engl J Med. 2014;371(10):906-917.

21. Palumbo A, Bringhen S, Petrucci MT, et al. Intermediate-dose melphalan improves survival of myeloma patients aged 50 to 70 : results of a randomized controlled trial. Blood. 2004;104(10):3052-3057. 
22. Palumbo A, Hajek R, Delforge M, et al; MM-015 Investigators. Continuous lenalidomide treatment for newly diagnosed multiple myeloma. N Engl J Med. 2012;366(19):1759-1769.

23. Waage A, Gimsing P, Fayers P, et al; Nordic MyelomaStudy Group. Melphalan and prednisone plus thalidomide or placebo in elderly patients with multiple myeloma. Blood. 2010;116(9):1405-1412.

24. Hulin C, Facon T, Rodon P, et al. Efficacy of melphalan and prednisone plus thalidomide in patients older than 75 years with newly diagnosed multiple myeloma: IFM 01/01 trial. J Clin Oncol. 2009;27(22):3664-3670.

25. Beksac M, Haznedar R, Firatli-Tuglular T, et al. Addition of thalidomide to oral melphalan/prednisone in patients with multiple myeloma not eligible for transplantation: results of a randomized trial from the Turkish Myeloma Study Group. Eur J Haematol. 2011;86(1):16-22.

26. Wijermans P, Schaafsma M, Termorshuizen F, et al; Dutch-Belgium Cooperative Group HOVON. Phase III study of the value of thalidomide added to melphalan plus prednisone in elderly patients with newly diagnosed multiple myeloma: the HOVON 49 Study. J Clin Oncol. 2010; 28(19):3160-3166.

27. Facon T, Mary JY, Hulin C, et al; Intergroupe Francophone du Myélome. Melphalan and prednisone plus thalidomide versus melphalan and prednisone alone or reduced-intensity autologous stem cell transplantation in elderly patients with multiple myeloma (IFM 99-06): a randomized trial. Lancet. 2007;370(9594):1209-1218.

28. Ludwig H, Hajek R, Tóthová E, et al. Thalidomide-dexamethasion compared with melphalan-prednisolone in elderly patients with multiple myeloma. Blood. 2009;113(15):3435-3442.

29. Niesvizky R, Flinn IW, Rifkin R, et al. Community based phase IIIB trial of three UPFRONT bortezomib-based myeloma regimens. J Clin Oncol. 2015;33(33):3921-3929.

30. Palumbo A, Bringhen S, Larocca A, et al. Bortezomib-melphalanprednisone-thalidomide followed by maintenance with bortezomibthalidomide compared with bortezomib-melphalan-prednisone for initial treatment of multiple myeloma: updated follow-up and improved survival. J Clin Oncol. 2014;32(7):634-640.
31. Stewart AK, Jacobus S, Fonseca R, et al. Melphalan, prednisone, and thalidomide vs melphalan, prednisone, and lenalidomide (ECOG E1A06) in untreated multiple myeloma. Blood. 2015;126(11):1294-1301.

32. Zweegman S, van der Holt B, Mellqvist UH, et al. Melphalan, prednisone, and lenalidomide versus melphalan, prednisone, and thalidomide in untreated multiple myeloma. Blood. 2016;127(9):1109-1116.

33. Magarotto V, Bringhen S, Offidani M, et al. Triplet vs doublet lenalidomide-containing regimens for the treatment of elderly patients with newly diagnosed multiple myeloma. Blood. 2016;127(9):1102-1108.

34. Fayers PM, Palumbo A, Hulin C, et al; Nordic Myeloma Study Group; Italian Multiple Myeloma Network; Turkish Myeloma Study Group; Hemato-Oncologie voor Volwassenen Nederland; Intergroupe Francophone du Myélome; European Myeloma Network. Thalidomide for previously untreated elderly patients with multiple myeloma: metaanalysis of 1,685 individual patient data from 6 randomized clinical trials. Blood. 2011;118(5):1239-1247.

35. Greco T, Edefonti V, Biondi-Zoccai G, et al. A multilevel approach to network meta-analysis within a frequentist framework. Contemp Clin Trials. 2015;42:51-59.

36. Zhu GQ, Shi KQ, Huang GQ, et al. A network meta-analysis of the efficacy and side effects of UDCA-based therapies for primary sclerosing cholangitis. Oncotarget. 2015;6(29):26757-26769.

37. TMai R, Zhou S, Zhong W, et al. Therapeutic efficacy of combined BRAF and MEK inhibition in metastatic melanoma: a comprehensive network meta-analysis of randomized controlled trials. Oncotarget. 2015;6(29):28502-28512.

38. Mhaskar R, Redzepovic J, Wheatley K, et al. Bisphosphonates in multiple myeloma: a network meta-analysis. Cochrane Database Syst Rev. 2012;5:CD003188.

39. Krstevska SB, Sotirova T, Balkanov T, Genadieva-Stavric S. Treatment approach of nontransplant patients with multiple myeloma. Mater Sociomed. 2014;26(5):348-351.

40. Cejalvo MJ, de la Rubia J. Clinical treatment of newly diagnosed multiple myeloma. Expert Rev Hematol. 2015;8(5):595-611.
OncoTargets and Therapy

\section{Publish your work in this journal}

OncoTargets and Therapy is an international, peer-reviewed, open access journal focusing on the pathological basis of all cancers, potential targets for therapy and treatment protocols employed to improve the management of cancer patients. The journal also focuses on the impact of management programs and new therapeutic agents and protocols on

\section{Dovepress}

patient perspectives such as quality of life, adherence and satisfaction The manuscript management system is completely online and includes a very quick and fair peer-review system, which is all easy to use. Visit http://www.dovepress.com/testimonials.php to read real quotes from published authors. 